\title{
Right-to-left shunt and migraine: the strength of the relationship
}

EA Wammes-van der Heijden ${ }^{1}$, CC Tijssen ${ }^{2} \&$ ACG Egberts ${ }^{1,3}$

${ }^{1}$ Utrecht Institute for Pharmaceutical Sciences, Utrecht, ${ }^{2}$ Department of Neurology, St Elisabeth Hospital Tilburg, and ${ }^{3}$ Hospital Pharmacy MiddenBrabant, TweeSteden Hospital and St Elisabeth Hospital Tilburg, the Netherlands

\section{Cephalalgia}

Wammes-van der Heijden EA, Tijssen CC \& Egberts ACG. Right-to-left shunt and migraine: the strength of the relationship. Cephalalgia 2006; 26:208-213. London. ISSN 0333-1024

Several studies have shown that the prevalence of a cardial right-to-left shunt (RLS) in patients with migraine with aura is significantly higher than in patients without migraine. To assess the strength of the possible relationship between RLS and migraine, the literature concerning this subject was systematically reviewed. We identified seven relevant studies. Among patients with RLS migraine with aura was 3.5 times more prevalent than among subjects without RLS [MantelHaenszel odds ratio $\left(\mathrm{OR}_{\mathrm{MH}}\right) 3.5 ; 95 \%$ confidence interval (CI) 2.1, 5.8]. In patients with ischaemic stroke migraine was more than two times more prevalent in patients with RLS than in patients without RLS $\left(\mathrm{OR}_{\mathrm{MH}} 2.1 ; 95 \% \mathrm{CI} 1.6,2.9\right)$. Our review shows that there is a clear association between RLS and migraine, especially migraine with aura. The relationship between RLS and migraine is further substantiated by the observations of disappearance and improvement of migraine symptoms after closure of the foramen ovale. However, the mechanism as well as the question about causality of this association has to be further elucidated. $\square$ Migraine, patent foramen ovale, right-to-left shunt, stroke

Prof. Dr A. C. G. Egberts, Department of Pharmacoepidemiology and Pharmacotherapy, Utrecht Institute for Pharmaceutical Sciences, PO Box 80082, 3508 TB Utrecht, The Netherlands. Tel. + 3130253 7324, fax + 3130253 9166, e-mail a.c.g.egberts@pharm.uu.nl Received 23 November 2004, accepted 15 May 2005

\section{Introduction}

A recent meta-analysis showed that interatrial septal abnormalities-patent foramen ovale (PFO) and atrial septal aneurysm (ASA) - are risk factors for ischaemic stroke (IS) in patients younger than 55 years (1). PFO, with or without ASA, may cause right-to-left shunting of blood. Recently it was found that the prevalence of a cardial right-to-left shunt (RLS) in patients with migraine with aura (MA), another risk factor for IS in the young, was about 2.5 times higher compared with a group of patients without migraine $(2,3)$ and that this association between RLS and MA is independent of sex (4). This higher prevalence of RLS may, at least partly, explain the increased risk for IS in patients with MA, as first postulated by Ries et al. in 1996 (5).

We systematically reviewed the available literature to quantify the strength of the relationship between RLS and migraine in patients with and without IS.

\section{Methods}

A Medline search (accessed through Pubmed, most recent search April 2005) was performed for the words (all fields) patent foramen ovale, PFO, cardiac abnormalities, right-to-left shunt, RLS, atrial septal aneurysm or atrial septal defect and migraine. Other papers of potential interest were sought from the reference lists of the retrieved papers. Only studies with quantitative data about the relationship RLS-migraine were included. There were no restrictions with respect to the design and quality of the studies. Unpublished data were not sought. We evaluated the strength of the association between RLS as the determinant (independent variable) and migraine as the outcome (dependent variable). Since RLS is a risk factor for IS, we stratified the results for patients with IS and patients without IS. Mantel-Haenszel odds ratios $\left(\mathrm{OR}_{\mathrm{MH}}\right)$ with $95 \%$ confidence intervals ( $95 \% \mathrm{CI}$ ) of pooled data were calculated. 


\section{Results}

The Medline search identified 76 papers. Crossreferencing did not result in additional papers. Of these, seven studies provided quantitative data concerning the relation between RLS and migraine. One of these studies (6) appeared to be an extension of an earlier study, so the data of this latest study were used (see also Table 1). The investigators of these reports studied the relationship between RLS and migraine in cross-sectional studies either by determining the prevalence of migraine in samples of patients with and without RLS $(7,8)$ or by determining the prevalence of RLS in patients with and without migraine $(2,3,6,9,10)$.

\section{Patients without IS}

In a consecutive unselected cohort of migraine patients a prevalence of RLS of $48 \%$ (54/113) was found in patients with MA compared with 23\% (12/ $53)$ in patients with migraine without aura (MoA) and $20 \%(5 / 25)$ in control subjects (age-matched nonmigraine members of the hospital staff) (3). Del Sette et al. found a prevalence of RLS of $40 \%$ in 80 consecutive patients with MA compared with $16 \%$ in 50 healthy control subjects without migraine $(2,6)$. Domitrz et al. found a prevalence of RLS of 53\% (33/ $62)$ in patients with MA compared with $25 \%(15 / 60)$ in patients with MoA and 25\% (16/65) in healthy age-matched controls (10). These three studies consistently show that, in patients without IS, MA was more prevalent $\left(\mathrm{OR}_{\mathrm{MH}} 3.5 ; 95 \% \mathrm{CI} 2.1,5.8\right)$ in patients with RLS than in those without RLS (Table 1).

\section{Patients with IS}

Using data from a prospective stroke registry to determine the characteristics of acute IS in patients with active migraine, it was shown that patients with first-ever IS and migraine (with and without aura)

Table 1 Patients without ischaemic stroke

\begin{tabular}{lccl}
\hline Study & RLS+ & RLS- & Odds ratio $(95 \% \mathrm{CI})$ \\
\hline Anzola 1999 & $54 / 59$ & $59 / 79$ & $3.7(1.3,10.4)$ \\
Angeli 2001* & $32 / 40$ & $48 / 90$ & $3.5(1.5,8.4)$ \\
Domitrz 2004 & $33 / 49$ & $29 / 78$ & $3.5(1.6,7.4)$ \\
Total & $119 / 148$ & $136 / 247$ & OR $_{\mathrm{MH}}=3.5(2.1,5.8)$ \\
\hline
\end{tabular}

Comparison: right-to-left shunt (RLS) vs. no RLS.

Outcome: migraine with aura (vs. no migraine).

*Part of the results of this study were formerly published: Del Sette 1998 (2). had a RLS almost twice as often as patients with firstever IS without migraine: $18.5 \%(24 / 130)$ compared with $10.1 \%$ (121/1195) (9). In 581 patients (1855 years) with a cryptogenic stroke, $27.3 \%$ of the patients with a PFO had migraine (with and without aura) compared with $14.0 \%$ of the patients without a PFO (7). In a series of 74 consecutive patients presenting with an acute stroke of undetermined origin, PFO was found in 44 of 74 patients, of whom $36 \%$ $(16 / 44)$ had MA, compared with $13 \%(4 / 30)$ of the patients without PFO (8). This difference was not observed for MoA. The combined results of these three studies show that in patients with IS, migraine (with and without aura) was twice as prevalent $\left(\mathrm{OR}_{\mathrm{MH}} 2.1 ; 95 \% \mathrm{CI} 1.6,2.9\right)$ in patients with RLS as in patients without RLS (Table 2).

\section{Discussion}

Among patients with RLS, MA is clearly more prevalent than among persons without RLS. The prevalence of RLS found in the control groups without migraine is comparable to the prevalence of $\mathrm{PFO}$ in the general population, i.e. about $25 \%$ (11).

Several limitations of our analysis should be mentioned. First, the applied diagnostic procedures and diagnostic criteria for PFO, ASA or RLS were not the same in all studies, which may explain the differences between studies in the reported prevalence of RLS. Transcranial Doppler sonography with contrast medium (cTCD) $(2,3,6,10)$, transoesophageal echocardiography (TEE) with contrast medium (gold standard) (7), transthoracic echocardiogram (TTE) with contrast medium or a combination of these techniques $(8,9)$ were used to diagnose cardiac abnormalities. Although this may have influenced the absolute prevalence of interatrial septal abnormalities found in each individual study, this does not influence the relative risk estimates (OR), as within each study there were no diagnostic differences between the various groups of patients that were compared. Bias towards a positive finding of

Table 2 Patients with ischaemic stroke

\begin{tabular}{lccl}
\hline Study & RLS+ & RLS- & Odds ratio $(95 \%$ CI) \\
\hline Milhaud 2001 & $24 / 145$ & $106 / 1180$ & $2.0(1.2,3.3)$ \\
Lamy 2002 & $73 / 267$ & $44 / 314$ & $2.3(1.5,3.5)$ \\
Sztajzel 2002 & $27 / 44$ & $14 / 30$ & $1.8(0.7,4.6)$ \\
Total & $124 / 456$ & $164 / 1524$ & OR $_{\mathrm{MH}}=2.1(1.6,2.9)$ \\
\hline
\end{tabular}

Comparison: RLS vs. no RLS.

Outcome: migraine (vs. no migraine). 
migraine was unlikely since, in both studies which determined the prevalence of migraine (outcome) in patients with and without RLS, a blinding procedure (7) or independent observers (8) were used to establish the diagnosis. Although migraine was diagnosed according to the criteria of the International Headache Society in all studies, misclassification with respect to migraine cannot be ruled out. Besides, in two of the three studies with IS patients no distinction was made between MA and MoA (7, 9). This might explain the lower odds ratio for the relationship between RLS and migraine found in IS patients, since up to now no relationship between RLS and MoA has been established $(3,8,10,12)$. Second, we did not put restrictions on the design and quality criteria of the studies, since there were only a few studies with quantitative data. Third, the studies included in our analysis were relatively small and were all non-randomized. Within both subgroups, the odds ratios of the individual studies were, however, remarkably consistent. Fourth, given the observational design of the included studies and the fact that it was not possible to take into account the presence of thromboembolic risk factors in our analysis, we can not eliminate confounding and therefore could not state that the found association is causal in nature. However, in five of six studies the age, a major confounder, of the cases were comparable to the ages of control subjects $(2,6,8-10)$. Furthermore, the study of Lamy et al. showed that patients with PFO were less likely to have traditional risk factors of stroke than patients without PFO (7). This means that in case the prevalence of migraine was higher in patients with these risk factors, the found association between RLS and migraine would be even stronger. Finally, we sought only published data, which due to publication bias may have overestimated our results. Despite these limitations, we believe that an association between RLS and MA does exist.

Further evidence that substantiates the relationship between RLS and migraine is found in three patient groups. First, in patients with cerebral autosomal dominant arteriopathy with subcortical infarcts and leukoencephalopathy (CADASIL), Angeli et al. found a prevalence of RLS of $80 \%$ (4/5) (6). All patients with CADASIL and migraine (4/4) showed RLS. However, this concerns a report of only one Italian family and needs confirmation.

Second, in a group of divers with decompression illness, MA in daily life unconnected with diving was found in $47.5 \%$ of divers with a large RLS at rest and $13.8 \%$ of divers with no shunt (12). The prevalence of MoA was the same in both groups. In divers with RLS, decompression illness is probably caused by paradoxical gas embolism (13).

Finally, though all based on uncontrolled studies, the observation of improvement or even disappearance of migraine after closure of the PFO supports the association between RLS and migraine (Table 3 ) $(8,14-20)$. Based on their design, these studies have several limitations which have to be mentioned. First of all, there can be a clear placebo effect in migraine treatment. However, in two studies patients were unaware of a potential benefit of the intervention on migraine outcome $(15,18)$. Furthermore, the study of Schwerzmann et al. showed no reduction of nonmigrainous headaches (15). Although a placebo effect cannot be excluded, it is unlikely to have produced such consistent results. Second, all data on headache frequency before the closure procedure were retrospective, which can introduce recall bias. Finally, most of the patients received antiplatelet therapy after the closure procedure for a period of 6 months. However, the mean follow-up time of these patients was $\geq 12$ months and sustained migraine relief was observed after antiplatelet therapy was discontinued. Additionally, it has to be mentioned that new-onset migraine has also occurred in patients (immediately) after closure of a PFO or an atrial septal defect (18, 20-22).

The underlying mechanism of the possible relation between RLS and migraine remains speculative. Wilmshurst et al. (18) postulated that RLS allows trigger substances in the venous circulation such as vasoactive chemicals and microemboli to bypass the pulmonary filter and reach the brain, inducing a migraine attack. Interestingly, paradoxical emboli seem to have a particular propensity for the posterior circulation (23), the area in which hypoperfusion occurs during the aura phase. It has also been mentioned that emboli may be formed within the atrial septal defect itself (24). Finally, it has been suggested that a particular genetic substrate might determine both atrial septal abnormalities and migraine $(2,7)$. Wilmshurst et al. showed that there is dominant inheritance of atrial shunts, which is linked to inheritance of MA in some families (25).

The question whether the risk of migraine varies with the degree of shunting is controversial. Such a relationship was found in persons with decompression illness (12), though was not observed in patients with a cryptogenic stroke; MA was found in $22.2 \%$, $29.2 \%$, and $28.1 \%$ of patients with small (three to nine microbubbles), moderate (10-30 microbubbles) and large (>30 microbubbles) shunts, respectively (7). 


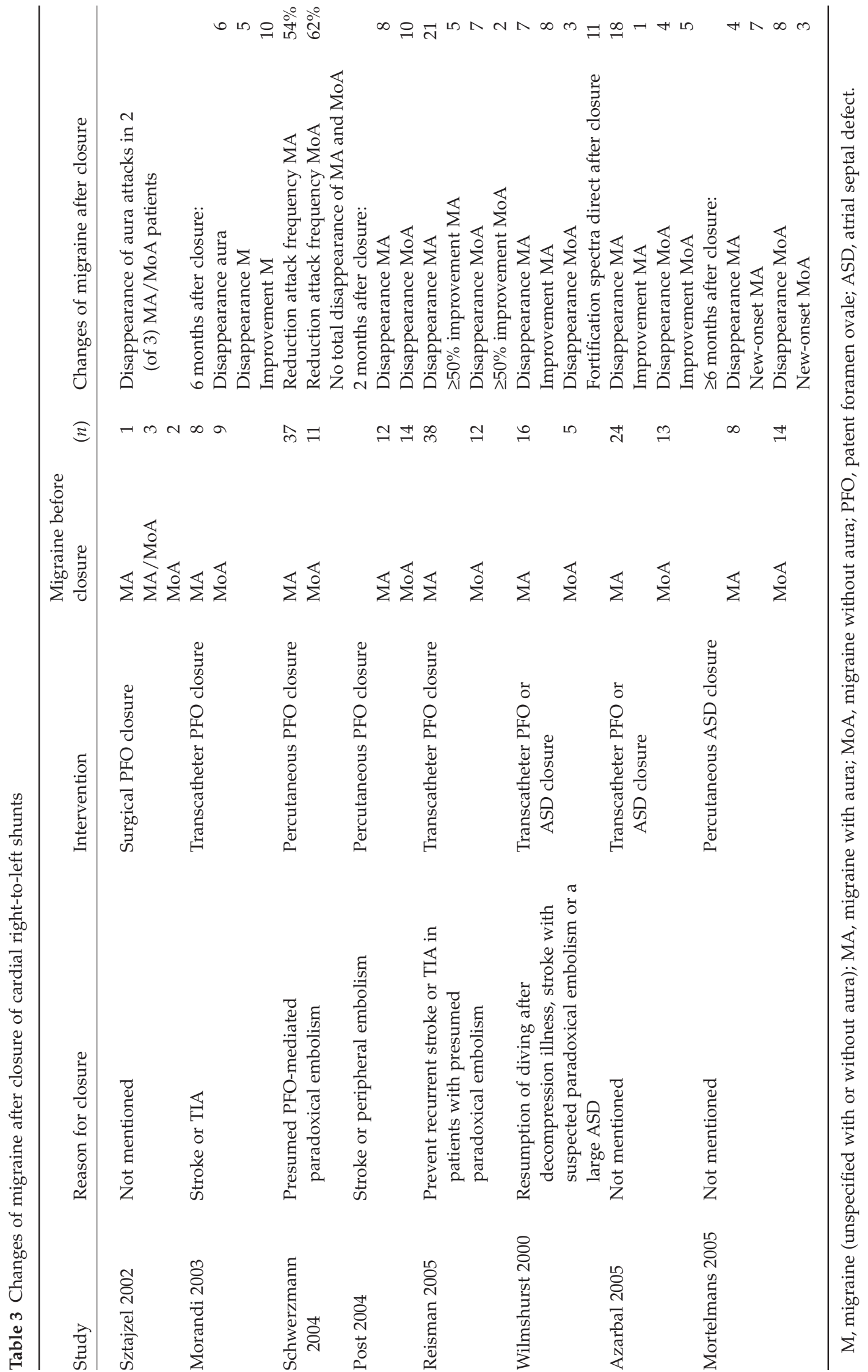




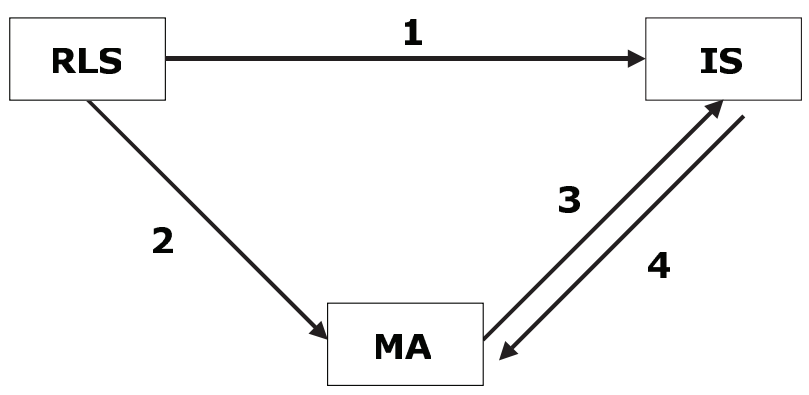

Figure 1 Relationships between right-to-left shunt (RLS), ischaemic stroke (IS) and migraine with aura (MA) as reported in the literature. 1. Interatrial septal abnormalities are potential risk factors for stroke in patients younger than 55 years (1). 2. Patients with RLS have a higher risk for having MA compared with normal controls $(3,6,8,10)$. The observation of improvement or even disappearance of migraine after closure of the RLS supports this relationship $(8,14-20)$. Wilmshurst et al. showed that there is dominant inheritance of atrial shunts, which is linked to inheritance of MA in some families (25). 3. MA is a risk factor for getting an ischaemic stroke (26). 4. Ischaemic events, for example caused by paradoxical embolization, might be a trigger for cortical spreading depression causing migrainous aura (18).

What does this all mean for the various relationships between RLS, MA and IS that have been reported in the literature (Fig. 1)? The studies that have shown that MA is a risk factor for IS (arrow 3) did not take the presence of RLS as a potential confounder into account. This means that RLS may explain at least a part of the increased ischaemic risk in patients with MA. In those patients arrow 3 can be replaced by the hypothesis that RLS may lead to subtle emboli entering the central circulation (arrow 1) and subsequently may trigger a migraine attack, especially MA (arrow 4), and arrow 2 will be crossed out.

In conclusion, the current evidence indicates that there is an association between RLS and MA. However, the mechanism, as well as the question whether this association is also causal in nature, have to be further elucidated. This knowledge may shed new light on the pathogenesis of migraine in special cases, possibly with new therapeutic options in the distant future.

\section{References}

1 Overell JR, Bone I, Lees KR. Interatrial septal abnormalities and stroke. A meta-analysis of case-control studies. Neurology 2000; 55:1172-9.

2 Del Sette M, Angeli S, Leandri M, Ferriero G, Bruzzone GL, Finocchi C, Gandolfo C. Migraine with aura and rightto-left shunt on transcranial Doppler: a case-control study. Cerebrovasc Dis 1998; 8:327-30.
3 Anzola GP, Magoni M, Guindani M, Rozzini L, Dalla Volta G. Potential source of cerebral embolism in migraine with aura: a transcranial Doppler study. Neurology 1999; 52: 1622-5.

4 Anzola GP, Del Sette M, Rozzini L, Zavarise P, Morandi $\mathrm{E}$, Gandolfo $\mathrm{C}$ et al. The migraine-PFO connection is independent of sex. Cerebrovasc Dis 2000; 10:163.

5 Ries S, Steinke W, Neff W, Schindlmayr C, Meairs S, Hennerici $M$. Ischemia-induced migraine from paradoxical cardioembolic stroke. Eur Neurol 1996; 36:76-8.

6 Angeli S, Carrera P, Del Sette M, Assini A, Grandis M, Biancolini D et al. Very high prevalence of right-to-left shunt on transcranial Doppler in an Italian family with cerebral autosomal dominant angiopathy with subcortical infarcts and leukoencephalopathy. Eur Neurol 2001; 46:198-201.

7 Lamy C, Giannesini C, Zuber M, Arquizan C, Meder JF, Trystram $\mathrm{D}$ et al. Clinical and imaging findings in cryptogenic stroke patients with and without patent foramen ovale. The PFO-ASA study. Stroke 2002; 33:706-11.

8 Sztajzel R, Genoud D, Roth S, Mermillod B, Le Floch-Rohr J. Patent foramen ovale, a possible cause of symptomatic migraine: a study of 74 patients with acute ischemic stroke. Cerebrovasc Dis 2002; 13:102-6.

9 Milhaud D, Bogousslavsky J, van Melle G, Liot P. Ischemic stroke and active migraine. Neurology 2001; 57:1805-11.

10 Domitrz I, Mieszkowski J, Kwieciński H. The prevalence of patent foramen ovale in patients with migraine. Neurol Neurochir Pol 2004; 38:89-92.

11 Hagen PT, Scholz PT, Edwards WD. Incidence and size of patent foramen ovale during the first 10 decades of life: an autopsy study of 965 normal hearts. Mayo Clin Proc 1984; 59:17-20.

12 Wilmshurst $\mathrm{P}$, Nightingale S. Relationship between migraine and cardiac and pulmonary right-to-left shunts. Clin Sci 2001; 100:215-20.

13 Schwerzmann M, Seiler C, Lipp E, Guzman R, Lövblad $\mathrm{KO}$, Kraus $\mathrm{M}$, Kucher N. Relation between directly detected patent foramen ovale and ischemic brain lesions in sport divers. Ann Intern Med 2001; 134:21-4.

14 Morandi E, Anzola GP, Angeli S, Melzi G, Onorato E. Transcatheter closure of patent foramen ovale: a new migraine treatment? J Interv Cardiol 2003; 16:39-42.

15 Schwerzmann M, Wiher S, Nedeltchev K, Mattle HP, Wahl A, Seiler C et al. Percutaneous closure of patent foramen ovale reduces frequency of migraine attacks. Neurology 2004; 62:1399-401.

16 Post MC, Thijs V, Herroelen L, Budts WIHL. Closure of a patent foramen ovale is associated with a decrease in prevalence of migraine. Neurology 2004; 62:1439-40.

17 Reisman M, Christofferson RD, Jesurum J, Olsen JV, Spencer MP, Krabill KA et al. Migraine headache relief after transcatheter closure of patent foramen ovale. JACC 2005; 45:493-5.

18 Wilmshurst PT, Nightingale S, Walsh KP, Morrison WL. Effect on migraine of closure of cardiac right-to-left shunts to prevent recurrence of decompression illness or stroke or for haemodynamic reasons. Lancet 2000; 356:1648-51.

19 Azarbal B, Tobis J, Suh W, Chan V, Dao C, Gaster R. Association of interatrial shunts and migraine headaches. JACC 2005; 45:489-92. 
20 Mortelmans K, Post M, Thijs V, Herroelen L, Budts W. The influence of percutaneous atrial septal defect closure on the occurrence of migraine. Eur Heart J 2005 March 3 [Epub ahead of publication].

21 Rodés-Cabau J, Molina C, Serrano-Munuera C, Casaldáliga J, Alvarez-Sabin J, Evangelista A, Soler-Soler J. Migraine with aura related to the percutaneous closure of an atrial septal defect. Catheter Cardiovasc Interv 2003; 60:540-2.

22 Yew G, Wilson NJ. Transcatheter atrial septal defect closure with the amplatzer septal occluder. Catheter Cardiovasc Interv 2005; 64:193-6.

23 Venketasubramanian N, Sacco RL, Di Tullio M, Sherman
D, Homma S, Mohr JP. Vascular distribution of paradoxical emboli by transcranial Doppler. Neurology 1993; 43:1533-5.

24 Pierangeli G, Cevoli S, Zanigni S, Sancisi E, Monaldini C, Donti A et al. The role of cardiac diseases in the comorbidity between migraine and stroke. Neurol Sci 2004; 25:S129_ S131.

25 Wilmshurst PT, Pearson MJ, Nightingale S, Walsh KP, Morrison WL. Inheritance of persistent foramen ovale and atrial septal defects and the relation to familial migraine with aura. Heart 2004; 90:1315-20.

26 Tietjen GE. The relationship of migraine and stroke. Neuroepidemiology 2000; 19:13-9. 